\title{
Buttonhole: provare o non provare?
}

\section{Luigi Tazza ${ }^{1}$, Nicola Panocchia ${ }^{1}$, Massimo Lodi ${ }^{2}$, Giuseppe Bonforte ${ }^{3}$, Patrizia Silvestri ${ }^{1}$, Luciano Carbonari ${ }^{4}$}

\author{
${ }^{1}$ U.O.S. Dialisi, Dipartimento di Scienze Chirurgiche, Università Cattolica Del Sacro Cuore, Roma \\ ${ }^{2}$ U.O.C. Nefrologia e Dialisi, Ospedale Spirito Santo, Pescara \\ ${ }^{3}$ E.O.C. Ospedale Beata Vergine, Mendrisio - Svizzera \\ ${ }^{4}$ S.O.D. Chirurgia Vascolare, Ospedali Riuniti, Ancona
}

ButTONHOLE TEChNiQUe: TO TRY OR NOT TO TRY?

Abstract. Starting from the recent analysis about the buttonhole technique (BT) by Dr. Napoli (6), our study focuses on some uncertain issues of the results. In particular, the increased risk of infection and the low incidence of aneurysms associated with BT. If BT safety will be confirmed in future studies it might be chosen for patients who present a particular arteriovenous fistula (AVF) location and in order to reduce aneurysm formation.

Key words: Vascular access, Buttonhole technique, Cannulation of arteriovenous fistula

Conflict of interest: None.

Financial support: None.

Accettato: 21 Febbraio 2014

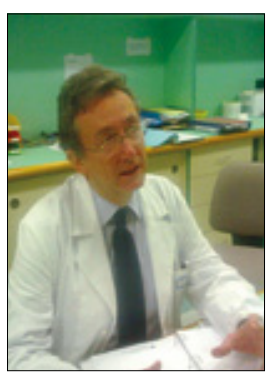

Luigi Tazza

\section{Introduzione}

Non c'è dubbio che, in tempi recenti, la BT (Buttonhole Technique, puntura ad asola) abbia avuto il merito di riportare l'attenzione di tutti gli operatori dell'emodialisi nel settore importante, ma un po' trascurato, delle tecniche di venipuntura $(1,2)$.

Il successo della FAV (Fistola Artero Venosa) dipende in gran parte da una corretta puntura; gli ottimi outcome di tale accesso, giustamente considerato il gold standard, sono legati prevalentemente alla venipuntura ad area (APT, area puncture technique) $\mathrm{o}$, in misura minore, a quella "a scala di corda"(RL, rope-ladder).

Tra le varie esperienze con BT, sono disponibili pochi studi randomizzati (3-5) e poche review. La recente analisi della letteratura realizzata da Napoli (6) è un buon punto di partenza, non per tentare la ricerca di ulteriori evidenze, ma per ridiscutere alcuni risultati già noti.

Il presente contributo è rivolto a coloro che sono soddisfatti della venipuntura ad area e che si chiedono cosa potrebbero avere in più da $\mathrm{BT}$ e a quale prezzo.

\section{Commento ai dati della letteratura}

Al momento, i dati esaminabili restano quelli descritti da
Napoli, anche se risulta che diversi centri abbiano introdotto da alcuni mesi la BT (un centro italiano da un anno ha applicato tale tecnica al $100 \%$ dei pazienti in trattamento) e si attendono nuovi report. Tutte le sei pubblicazioni in esame, 3 studi randomizzati e 3 review (7-9), risultano, a nostro giudizio, solo orientative, non permettendo di raggiungere particolari evidenze.

I diversi bias sono, al solito, la scarsa omogeneità dei parametri di studio e delle tecniche realmente messe a confronto (BT con o senza polycarbonate peg (3), ago smusso sì o no e modalità di disinfezione della cute, tanto per fare qualche esempio) e il modesto numero dei campioni esaminati. Anche quando, nel disegno, il sample size sembra adeguato, poi, nell'analisi "as treated", i numeri diventano esigui.

Raramente viene considerato un dettaglio critico, almeno per il rischio infettivo, come la tecnica di rimozione della crosta precedente all'incannulamento. Numerosi, poi, sono, nelle casistiche in esame, i fattori confondenti (uso di pomata anestetica o antibiotica e rotazione dello staff infermieristico per citarne solo alcuni).

Negli studi, inoltre, si esaminano insieme due popolazioni a mio giudizio diverse, le FAV di nuova istituzione e quelle già in uso da mesi, che meriterebbero confronti separati. Si aggiunga, poi, la breve durata degli studi, che si aggira su 12 mesi, troppo poco per confrontare alcuni parametri long term come la formazione di aneurismi e di stenosi e la sopravvivenza dell'accesso $(3,7,9)$. 


\section{Sicurezza della BT}

Chi decide di provare la BT deve essere convinto che la tecnica offra garanzie di sicurezza complessiva almeno pari all'APT/RL.

Da diverse pubblicazioni sappiamo che la BT è sicura per parecchi outcome (sopravvivenza, ospedalizzazioni) ma non per le complicanze infettive. Nelle review e in 2 su 3 studi randomizzati $(4,5)$ è stata osservata una maggiore incidenza di infezioni nella BT. La formazione mirata del canale venocutaneo rappresenta una fonte di annidamento dei microrganismi più difficile da disinfettare rispetto alla cute. È un po' come, secondo la riuscita descrizione di Napoli, trasformare una FAV in un catetere. Nell'ipotesi più ottimistica, possiamo aspettarci che la BT possa essere sicura nei riguardi del rischio infettivo come le tecniche usuali, a patto di raggiungere, con la disinfezione e l'uso ottimizzato della migliore rimozione sistematica della crosta (10), gli stessi risultati di abbattimento della carica batterica che si ottengono sulla cute e, per continuare il parallelismo, con un catetere venoso ottimamente gestito.

Sarà, quindi, necessario avvalersi di studi nuovi e completi, capaci di dare risultati chiari che confrontino le modalità di rimozione della crosta. Parimenti, sarà necessario avvalersi sempre delle più spinte tecniche di prevenzione delle infezioni già note $\mathrm{e}$, possibilmente, implementarle (10) (preventivo lavaggio dell'arto e doppia disinfezione, prima della rimozione della crosta e dopo).

\section{Risultati di durata e funzionamento dell'accesso con BT}

Un'ottimale sopravvivenza della FAV con BT, almeno nel breve periodo, sembra possibile in diversi studi $(3,7)$. Aspettiamo, con il tempo, più numerose e convincenti conferme di tale importante osservazione, ma occorrono periodi di followup adeguati. Molti sono, in letteratura, i giudizi positivi sulla BT, rispetto alle tecniche usuali, sia per quanto riguarda il numero dei ricoveri ospedalieri e dei reinterventi che per le caratteristiche di utilizzo (tempo necessario all'applicazione degli aghi, tempo di sanguinamento dopo la loro rimozione e comparsa di ematomi) e la qualità della vita, come il dolore alla puntura e l'aspetto estetico.

\section{Vantaggi ottenibili con la BT}

Da quanto finora detto potremo aspettarci dalla BT, al massimo, gli stessi ottimi risultati delle tecniche usuali.

Alcuni studi $(3,7)$ attribuiscono alla BT, rispetto alle tecniche usuali, una ridotta formazione di aneurismi de novo nella sede della puntura e un meno veloce accrescimento delle dimensioni degli aneurismi preesistenti alla BT. Riguardo alla genesi degli aneurismi sulle FAV, numerosi studi descrivono quella legata alle stenosi. In prossimità della stenosi, il flusso turbolento favorisce la formazione di una dilatazione della vena (11). Oltre al flusso turbolento, altri fattori di non facile determinazione possono indurre la dilatazione, tra i quali le proprietà strutturali della parete della vena, sia congenite che acquisite.

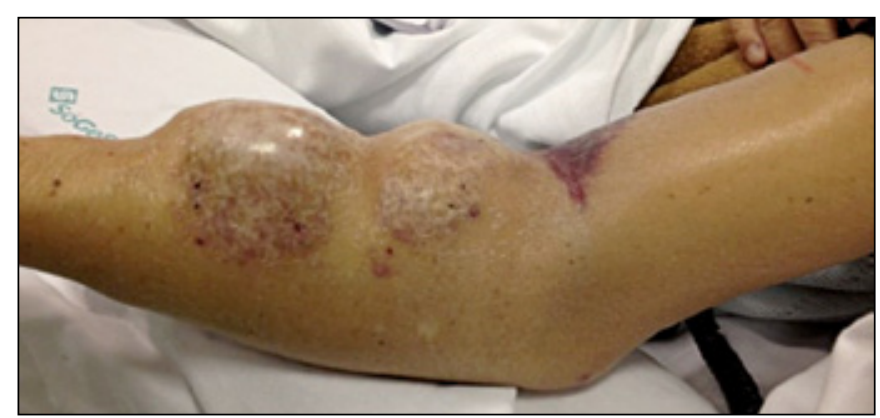

Fig. 1 - Atrofia cutanea cicatriziale sovrastante ampie dilatazioni aneurismatiche nelle sedi di puntura ad area.

Alcuni sostenitori della BT fanno notare, a nostro giudizio ragionevolmente, che l'instaurazione del canale venocutaneo, specialmente se ottenuta fin dal primo utilizzo della FAV, è in grado di preservare dal danno della venipuntura l'intera estensione della restante parete della FAV (1). Le tecniche usuali, dopo qualche tempo, trasformano ampi tratti delle aree di puntura in un campo di tagli a tutto spessore variamente riparati, di cui noi possiamo vedere le numerose cicatrici disseminate sulla cute (Fig. 1). Non dimentichiamo che gli aghi taglienti normalmente in uso, affilati e di grande diametro, durante un solo passaggio nei tessuti incontrano (e presumibilmente interrompono) un numero non trascurabile di fibre elastiche, la cui successiva riparazione tissutale condurrà a una restitutio ad integrum non necessariamente perfetta. È possibile che, oltre alle stenosi e alle turbolenze del flusso, almeno nelle sedi di applicazione degli aghi, il ripetuto e diffuso danno procurato dalla venipuntura con tagliente determini un indebolimento della parete venosa che conduce, in seguito, alla sua dilatazione aneurismatica, lo stesso fenomeno che osserviamo spesso nelle aree di puntura delle FAV protesiche, dove, ovviamente, mancano del tutto le fibre elastiche nella parete. Possiamo osservare comunemente aneurismi e ampie aree dilatate nei portatori di FAV usate da anni con APT/RL. Al contrario, chi conosce qualcuno dei rari pazienti che da anni usano la BT ha potuto conoscere FAV praticamente prive di dilatazioni aneurismatiche anche dopo anni. Nella BT è comune con il tempo assistere alla formazione, nella sede del canale venocutaneo, di un'introflessione della cute, solitamente descritta come "ombelicatura".

Diverse pubblicazioni $(3,12)$, per quanto, come già osservato, su follow-up di breve durata e con abbondanti disomogeneità, confermano, nella BT, una minore incidenza di dilatazioni e aneurismi rispetto alle tecniche usuali.

Con il passare degli anni cosa avverrà a livello del1'"ombelicatura"?

È esperienza comune che alcuni pazienti, specialmente brachitipi sovrappeso con vene scarsamente apprezzabili, presentino FAV dove la puntura a sito variabile trova disponibili solo due aree di limitate dimensioni e molto vicine tra loro. Per costoro, una buona BT "di necessità" potrebbe offrire migliori risultati rispetto ai tentativi ad area. 


\section{Possibilità di applicare la BT}

Quasi tutte le pubblicazioni enfatizzano la cura particolare richiesta nelle prime 2-3 settimane di BT per la formazione e il consolidamento del canale venocutaneo. Sono possibili varie tipologie di organizzazione del lavoro infermieristico: tutte richiedono profonda consapevolezza, unità di intenti, notevole spirito di collaborazione e buona volontà. Certamente, non tutti i centri potranno contare su un pool di esperti operatori disposti a sincronizzare i propri turni lavorativi con quelli del paziente prescelto per la BT, allo scopo di lasciare la creazione del canale venocutaneo nelle mani di un'unica persona, fino al momento in cui ogni infermiere potrà procedere all'applicazione degli aghi smussi.

\section{Conclusione: provare o no la BT?}

Le pubblicazioni disponibili offrono molte suggestioni, ma poche certezze. Soprattutto per il rischio di infezione sarà necessario aspettare ulteriori conferme su popolazioni e followup consistenti.

Riteniamo che anche altre caratteristiche del paziente, come la location della fistola e, forse, lo stato nutrizionale, andranno considerate per selezionare $\mathrm{i}$ soggetti che più di altri potrebbero avvalersi della BT.

La ridotta formazione di dilatazioni e aneurismi associata alla $\mathrm{BT}$ rispetto alle tecniche a sito variabile, se verrà confermata, potrà rappresentare uno dei principali obiettivi per l'applicazione di tale tecnica.

\section{Riassunto}

Partendo dalla recente analisi della letteratura sulla BT realizzata da Napoli (6), gli Autori approfondiscono alcuni elementi di incertezza relativi ai risultati, in particolare l'aumentato rischio di infezione e la ridotta formazione di aneurismi associati a tale tecnica. Se la sua sicurezza verrà confermata in ulteriori studi di ampia durata, la BT potrebbe essere indicata per pazienti con particolare location della FAV e per ridurre la formazione di aneurismi.

Parole chiave: Accesso Vascolare, Tecnica di puntura "buttonhole", Incannulamento della fistola arterovenosa

Dichiarazione di conflitto di interessi: Gli Autori dichiarano di non avere conflitto di interesse.

Contributi economici agli Autori: Gli Autori dichiarano di non aver ricevuto sponsorizzazioni economiche per la preparazione dell'articolo.

Indirizzo degli Autori:

Prof. Luigi Tazza

U.O.S. Dialisi - Dipartimento di Scienze Chirurgiche

Università Cattolica del Sacro Cuore

00168 Roma

luigi.tazza@gmail.com

\section{Bibliografia}

1. Twardowski ZJ, Kubara H. Different sites versus constant sites of needle insertion into arteriovenous fistulas for treatment by repeated dialysis. Dial Transplant 1979; 8: 978-80.

2. Ball L, Treat L, Riffle V, et al. A multi-center perspective of the buttonhole technique in the Pacific Northwest. Nephrol Nurs J 2007; 34: 234-41.

3. Vaux E, King J, Lloyd S, et al. Effect of buttonhole cannulation with a polycarbonate PEG on in-center hemodialysis fistula outcomes: a randomized controlled trial. Am J Kidney Dis 2013; 62 (1): 81-8.

4. Chow J, Rayment G, San Miguel S, Gilbert M. A randomised controlled trial of buttonhole cannulation for the prevention of fistula access complications. J Ren Care 2011; 37 (2): 85-93.

5. MacRae JM, Ahmed SB, Atkar R, Hemmelgarn BR. A randomized trial comparing buttonhole with rope ladder needling in conventional hemodialysis patients. Clin J Am Soc Nephrol 2012; 7 (10): 1632-8.

6. Napoli M. Il buttonhole: come trasformare una fistola in un catetere. Giornale di Tecniche Nefrologiche \& Dialitiche 2013; 25 (4): 290-2.

7. Grudzinski A, Mendelssohn D, Pierratos A, Nesrallah G. A systematic review of buttonhole cannulation practices and outcomes. Semin Dial 2013; 26 (4): 465-75.

8. Mustafa RA, Zimmerman D, Rioux JP, et al. Vascular access for intensive maintenance hemodialysis: a systematic review for a Canadian Society of Nephrology clinical practice guideline. Am J Kidney Dis 2013; 62 (1): 112-31.

9. Atkar RK, Macrae JM. The buttonhole technique for fistula cannulation: pros and cons. Curr Opin Nephrol Hypertens 2013; 22 (6): 629-36.

10. Labriola L, Crott R, Desmet C, André G, Jadoul M. Infectious complications following conversion to buttonhole cannulation af native arteriovenous fistulas: a quality improvement report. Am J Kidney Dis 2011; 57 (3): 442-8.

11. Roy-Chaudhury P, Kelly BS, Zhang J, et al. Hemodialysis vascular access dysfunction: from pathophysiology to novel therapies. Blood Purif 2003; 21: 99-110.

12. Ball LK. Improving arteriovenous fistula cannulation skills. Nephrol Nurs J 2005; 32: 611-7. 